\title{
3D-Printed Pacifier-Shaped Mouthpiece for fMRI-Compatible Gustometers
}

\author{
(DDavid Munoz Tord, ${ }^{1,2,3, *}$ Géraldine Coppin, ${ }^{1,3, *}$ Eva R. Pool, ${ }^{1,2}$ Christophe Mermoud, ${ }^{1}$ Zoltan Pataky, ${ }^{4}$ \\ David Sander, ${ }^{1,2}$ and DSylvain Delplanque ${ }^{1,2}$
}

https://doi.org/10.1523/ENEURO.0208-21.2021

${ }^{1}$ Swiss Center for Affective Sciences, University of Geneva, 1202 Geneva, Switzerland, ${ }^{2}$ Department of Psychology, University of Geneva, 1205 Geneva, Switzerland, ${ }^{3}$ Department of Psychology, UniDistance Suisse, 3900 Brig, Switzerland, and ${ }^{4}$ Department of Medicine, University of Geneva, 1205 Geneva, Switzerland

\begin{abstract}
Gustometers have made it possible to deliver liquids in functional magnetic resonance imaging (fMRI) settings for decades, and mouthpieces are a critical part of these taste delivery systems. Here, we propose an innovative 3D-printed fMRI mouthpiece inspired by children's pacifiers, allowing human participants to swallow while lying down in an MRI scanner. We used a large sample to validate the effectiveness of our method. The results suggest that the mouthpiece can be used to deliver taste stimuli by showing significant clusters of activation in the insular and piriform cortex, which are regions that have been consistently identified in taste processing. This mouthpiece fulfills several criteria guaranteeing a gustatory stimulus of quality, making the delivery precise and reliable. Moreover, this new pacifier-shaped design is simple and cheap to manufacture, hygienic, comfortable to keep in the mouth, and flexible to use in diverse cases. We hope that this new method will promote and facilitate the study of taste and flavor perception in the context of reward processing in affective neuroscience, and thus, help provide an integrative approach to the study of the emotional nature of rewards.
\end{abstract}

Key words: 3D printing; flavor; gustometer; mouthpiece; fMRl; taste

\section{Significance Statement}

The neuronal networks underlying taste perception have been of great interest in the investigation of fundamental processes, as well as the investigation of the mechanisms involved in a variety of eating disorders. However, the study of food rewards requires specific equipment, combining both precision and comfort. Here, we provide a design for a customizable, fMRI-compatible mouthpiece capable of delivering different liquids in a precise and consistent manner to participants while lying down in a scanner. Additionally, this new pacifier shaped design is comfortable in the mouth and allows for the correction of imaging artifacts when combined with appropriate methods. This open-source design can be used to customize and manufacture mouthpieces to meet unique demands of specific research projects and individual needs.

\section{Introduction}

Studying the neuronal pathways of chemical senses (i.e., olfaction and gustation) requires special equipment. It is relatively easy to make olfactometers (Coppin, 2020), and the same statement may be even truer for gustometers (Canna et al., 2019). The gustometer is a tool specifically designed to deliver liquids.

Received May 10, 2021; accepted August 30, 2021; First published September 22, 2021.

The authors declare no competing financial interests.
Some gustometers have been used for almost 20 years (O'Doherty et al., 2002; Small et al., 2003). However, while mouthpieces, which are a critical part of the gustatory delivery system (Andersen et al., 2019; Canna et al., 2019), have not been updated in that time, the number of publications on the topic has kept increasing over the years.

Author contributions: G.C., E.R.P., C.M., D.S., and S.D. designed research; G.C. performed research; C.M., Z.P., and D.S. contributed unpublished reagents/analytic tools; D.M.T. and E.R.P. analyzed data; D.M.T., G.C., and S.D. wrote the paper. 


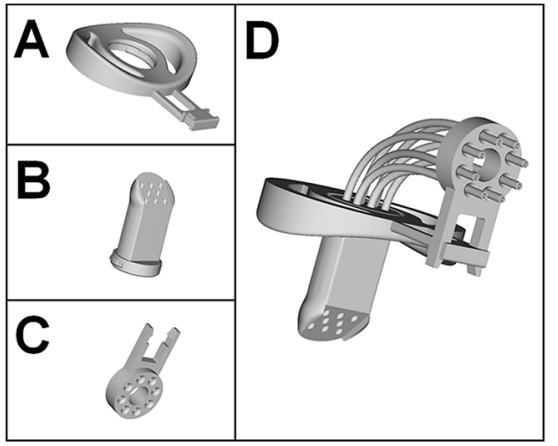

Figure 1. 3D representation of the fMRI-compatible mouthpiece. Detailed 3D representation of $(\boldsymbol{A})$ the mouth shield, $(\boldsymbol{B})$ the mouthpiece, $(\boldsymbol{C})$ the tube guide, and $(\boldsymbol{D})$ the complete mouthpiece assembled with eight tubes.

Here, we propose an innovative 3D-printed functional magnetic resonance imaging (fMRI)-compatible mouthpiece, which fulfills several criteria for a quality gustatory stimulus. First, this new mouthpiece (Fig. 1) allows participants to swallow liquids while lying down in a scanner, with their heads immobilized in a given position, and can remain comfortably in the mouth for a considerable amount of time without requiring any particular effort. Indeed, this design, inspired by children's pacifiers, replaces biting sticks which are sometimes used, onto which participants need to apply pressure with their teeth. Moreover, with biting sticks, it is sometimes necessary to take into account individual dental impressions (Goto et al., 2015). Second, up to eight different liquids can be delivered with this mouthpiece in a precise and consistent manner, making it possible to minimize somatosensory variations and allowing researchers to target the same taste buds over each repetition.

\section{Mouthpiece description}

The mouthpiece, inspired by children's pacifiers, consists of three parts: a mouth shield, an elongated teat, and a tube guide. These three pieces are separately printed using natural polylactic acid (PLA), a biodegradable plastic made from corn. Other plastics can be used, but it remains the researcher's responsibility to comply with the

This work was supported by the Firmenich SA Research Grant UN9046. ${ }^{*}$ D.M.T. and G.C. contributed equally to this work.

Acknowledgements: We thank Alain Hugon for his major contribution in the early stages of the design of the pacifier-shaped mouthpiece, Dr. Vanessa Sennwald for her insightful comments on this manuscript, Asli Erdemli for her useful comments on the data acquisition, and Lavinia Wuensch for her work on the data preprocessing. We also thank all the people from the Brain and Behavior Lab as well as from the Perception and Bioresponses Department of the Research and Development Division of Firmenich SA for their precious advice and their theoretical and technical competence.

Correspondence should be addressed to David Munoz Tord at david. munoztord@unige.ch.

https://doi.org/10.1523/ENEURO.0208-21.2021

Copyright (C) 2021 Munoz Tord et al.

This is an open-access article distributed under the terms of the Creative Commons Attribution 4.0 International license, which permits unrestricted use, distribution and reproduction in any medium provided that the original work is properly attributed. health standards of the country in which they are conducting experiments with this mouthpiece.

An oval mouth shield (Fig. 1A), with a curvature adapted to the morphology of the face, holds the mouthpiece comfortably on the lips. A cylindrical teat $(40 \mathrm{~mm}$ long $\times$ $22 \mathrm{~mm}$ in diameter) is inserted and clipped in the center of the mouth shield. This teat receives the tubes at one extremity and directs the liquids to the tongue at the other extremity (Fig. 1B). The part of the teat that goes into the mouth and is intended to come into contact with the tongue is beveled on one side and rounded on the other. This allows for an easy contact of the tongue with the teat to deliver drops of liquid comfortably and accurately. Depending on the research needs, up to eight tubes with an external diameter of $2.5 \mathrm{~mm}( \pm 0.3 \mathrm{~mm})$ can be inserted into the teat. The last piece is a tube guide (Fig. $1 C$ ) that is clipped onto the mouth shield and allows the tubes to be at a $90^{\circ}$ angle so that they run along the body of the participant lying on the MRI bed (Fig. 1D). The 3D printing files (stl) that we supply at https://github.com/munoztd0/ Mouthpiecegusto include seven versions with a diameter of $2.5 \pm 0.3 \mathrm{~mm}$ in steps of $0.1 \mathrm{~mm}$. All these versions make it possible to choose the parts that best fit together depending on the 2.5- $\mathrm{mm}$ tubes the researchers use and allows them to adjust the mouthpiece for different types of liquid or viscosity levels.

Since our plans are freely available, the mouthpiece can be made by any laboratory with access to a 3D printer, or it could otherwise be made by any 3D printing service company. It can be manufactured in quantity for a very low price ( 0.5 USD of material per piece). This makes it intrinsically hygienic, since each participant can get an individual mouthpiece. Moreover, the printing material can easily be adapted to comply with different countries' sanitary regulations. Our mouthpieces were made out of natural PLA, which is safe when used in contact with food (Conn et al., 1995). Finally, the mouthpiece does not require any modification to any preexisting apparatus and will seamlessly fit most gustometer setups.

\section{Materials and Methods}

\section{Participants}

This study was part of a larger experiment related to a different study question (NCT03347890) in which 97 right-handed participants were recruited. The experiment took place from 2018 to 2020 (i.e., before the COVID-19 pandemic). The study was approved by the Swissmedic ethical committee. All participants gave written informed consent and received 100 Swiss francs (the equivalent of 100 USD\$) for their participation in one session. In total, 12 participants were excluded from the analysis because of missing or incomplete data (five MRI and seven behavioral). We report data on the 85 remaining participants (55 female; mean age, $37.3 \pm 12.4$; min-max, 18-67 years). No predetermined sample size was estimated via statistical methods. All participants reported a normal sense of smell. All participants were asked to fast overnight because of the experiment occurring in the morning. 


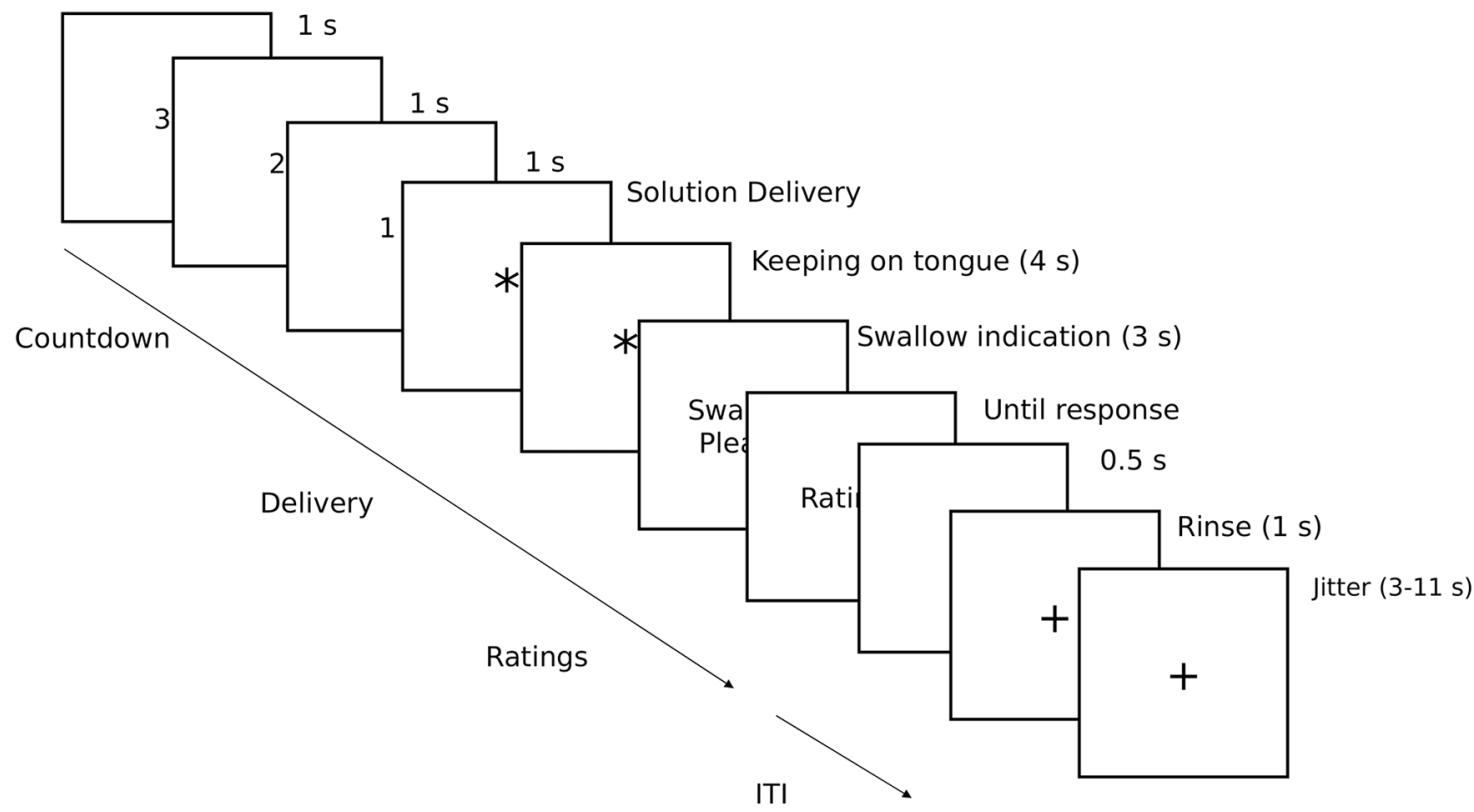

Figure 2. Task procedure. The sequence of the taste reactivity task, administered while participants were lying in the scanner. After a brief countdown, the participants were shown a fixation cross followed by an asterisk cueing the delivery of either a milkshake or a tasteless solution. They were asked to keep the solution on their tongue for $4 \mathrm{~s}$ and then prompted to swallow it. During this period, they were asked their perceived taste pleasantness and intensity of the solution. The experimental trials were intertwined with rinse trials to cleanse their palates.

\section{Stimuli preparations}

Milkshake preparations were made from a mix of milk $(300 \mathrm{~g})$ and ice cream $(60 \mathrm{~g})$ for a total of $71 \mathrm{kcal} / 100 \mathrm{~g}$. Potassium chloride $(\mathrm{KCl}, 1.8 \mathrm{~g})$ and sodium bicarbonate $\left(\mathrm{NaHCO}_{3}, 0.21 \mathrm{~g}\right)$ were diluted in 1 I of distilled water to recreate an artificial tasteless saliva solution. This main solution was then used to create less concentrated versions to be able to match each individual's perception of a tasteless solution. In total, there were four different tasteless concentrations $(1 / 1,3 / 4,1 / 2$, and $1 / 4)$ and three flavors of milkshake (strawberry, chocolate, and vanilla). We chose an individually adjusted tasteless solution as the control stimulus instead of plain water because water has been shown to have an inherent taste (Bartoshuk et al., 1964). The two solutions were taken out of the fridge simultaneously ( $30 \mathrm{~min}$ before the experiment) and delivered at ambient temperature. We took each participant's preferred milkshake flavor and the saliva solution that tasted the most neutral to them (i.e., closest to 50 on a scale from 0 to 100) as the two conditions for the experiment.

\section{Gustometer}

Single channel syringe pumps (Chemyx OEM) were used to achieve high flow control. Two syringes of up to $60 \mathrm{ml}$ were connected via 8-m-long food grade polyurethane tubing (external diameter $=4 \mathrm{~mm}$, inner diameter $=2.5 \mathrm{~mm}$ ) to a 1-m-long food grade polytetrafluoroethylene (PTFE) tubing (external diameter $=2.5 \mathrm{~mm}$, inner diameter $=1.9$ $\mathrm{mm}$ ) and to the mouthpiece at a delivery rate of $1 \mathrm{ml} / \mathrm{s}$. The syringe pumps were connected to a 16-port RS-232 rackmount device server (Moxa, Nport 5610) and then controlled via transmission control protocol (TCP) using specific $\mathrm{C}$ libraries designed for stimulus presentation software (MATLAB or python). Although it is out of the scope of this article, readers can refer to Andersen et al. (2019), Canna et al. (2019), or lannilli et al. (2015) for detailed instructions on how to set up an fMRI-compatible gustometer.

\section{Taste reactivity task}

A taste reactivity task was administered while participants were lying in the scanner. The task consisted in the evaluation of the perceived pleasantness and intensity of two different stimuli: a milkshake and a tasteless solution. During each trial, $1 \mathrm{ml}$ of the solution was administered, and the delivery order of the two conditions was randomized within each participant. Participants were visually guided through the task with on-screen indications. First, they saw a 3-s countdown before the solution delivery, followed by an asterisk indicating to keep the solution on their tongue until they saw the swallow indication "swallow please" (Fig. 2). We asked them to wait $4 \mathrm{~s}$ before swallowing to avoid adding movement noise to the blood oxygen level-dependent (BOLD) response. Since they were lying down, the mouthpiece was placed in a such a way that the solution was delivered at the center of the participant's tongue, and we expected that the solution would slide down to the back of their tongue in the 4-s gustation period. The experimental trials were intertwined with rinse trials to cleanse the participants' palates with 1 $\mathrm{ml}$ of water. All 40 evaluations (20 per solution) were done on visual analog scales displayed on a computer screen. Participants had to answer through a button-box placed 
in their hand. The visual scales for the intensity report ranged from "not perceived" to "extremely intense"; and from "extremely unpleasant" to "extremely pleasant" for liking ratings.

\section{Data acquisition}

The collection of the responses was controlled by a computer running MATLAB (version R2015a; MathWorks). The presentation of the stimuli was implemented using Psychtoolbox (version 3.0; Brainard, 1997). The acquisition of the neuroimaging data were performed via a 3-Tesla MRI system (Magnetom Tim Trio, Siemens Medical Solutions) supplied with a 32-channel head coil following a gradient echo (GRE) sequence to record BOLD signal. We recorded forty echoplanar imaging (EPI) slices per scan with an isotropic voxel size of $3 \mathrm{~mm}$. Our scanner parameters were set at: echo time $(T E)=20 \mathrm{~ms}$, repetition time $(T R)=2000 \mathrm{~ms}$, field of view $(F O V)=210 \times 210 \times 144 \mathrm{~mm}$, matrix size $=70 \times 70$ voxels, flip angle $=85^{\circ}, 0.6-\mathrm{mm}$ gap between slices. Structural whole brain T1-weighted $\left(T 1_{w}\right)$ images (isotropic voxel size $=1.0 \mathrm{~mm}$ ) were acquired, as well as dual gradient $B_{0}$ field maps (Fmaps) for each participant to correct for inhomogeneity distortions in the static-field.

\section{Preprocessing}

We combined the fMRI of the Brain (FMRIB) Software Library (FSL, version 4.1; Jenkinson et al., 2012) with the Advanced Normalization Tools (ANTS, version 2.1; Avants et al., 2011) to create a pipeline optimized for the preprocessing of our neuroimaging data.

A challenge of $\mathrm{AMRI}$ gustometry is that the BOLD signal is highly prone to movement artifacts, and thus, the swallowing of liquid solutions while lying down produces significant deglutition artifacts. To offset this loss of signalto-noise ratio (SNR), we followed Griffanti et al. (2017) a rigorous protocol based on an $\mathrm{fMRI}$ independent component analysis (ICA) to remove artifacts.

We used the multivariate exploratory linear optimized decomposition tool (MELODIC; Beckmann and Smith, 2004) to decompose our raw BOLD signal into independent components (ICs). The ICA-based strategy for motion artifact removal has been shown to be more reliable to remove motion-induced signal variations than regressions from motion parameters (Pruim et al., 2015). Two researchers independently hand classified a sample of 20 participants' IC into two categories: clear artifact (e.g., motion/deglutition, susceptibility, or blood flow in arteries) or potential signal. The categorizations were then compared between the two judges, and each discrepancy was discussed until an agreement was reached (interrater reliability $=93 \%$ ). The manually classified components obtained by this process were used to train a classifier using a random forest machine learning algorithm (Breiman, 2001). Leave-one-out testing, where we iteratively left one participant out of the training data and tested the classifier accuracy on the left-out participant, at the optimal sensitivity (threshold $=5$ ) resulted in a median $94 \%$ true positive rate (i.e., the percentage of true signal accurately classified). Consequently, we applied the FMRIB's ICA-based X-noiseifier (FIX) to automatize the denoising of our BOLD signal (Salimi-Khorshidi et al., 2014).

Field maps were then applied to correct geometric distortions and ANTS was used for a diffeomorphic co-registration of the preprocessed functional and structural images in the Montreal Neurologic Institute (MNI) space, using the nearest-neighbor interpolation and leaving the functional images in their native resolution. Finally, we applied a spatial smoothing of 8-mm full width half maximum (FWHM).

\section{Data analysis}

Statistical analyses of the behavioral data were performed with $\mathrm{R}$ (version 4.0; R Core Team, 2019). We report Cohen's $d_{z}$ and the $95 \%$ confidence intervals (Cls) as estimates of effect sizes for the paired $t$ tests (Lakens, 2013), as well as a Bayes factor $\left(B F_{10}\right)$ quantifying the likelihood of the data under the alternative hypothesis relative to the null hypothesis (Morey et al., 2015).

The Statistical Parametric Mapping software (SPM; version 12; Penny et al., 2011) was used to perform a random-effects univariate analysis on the voxels of the image time series following a two-stage approach to partition model residuals to take into account within-participant and between-participant variance (Holmes and Friston, 1988; Mumford and Poldrack, 2007).

We specified a subject-level general linear model (GLM) for each participant and added a high-pass filter cutoff of $1 / 128 \mathrm{~Hz}$ to eliminate possible low-frequency confounds (Talmi et al., 2008). Each regressor of interest was derived from the onsets and duration of the stimuli and was convoluted with a canonical hemodynamic response function (HRF) into the GLM to obtain weighted parameter estimates $(\beta)$. The subject-level GLM consisted of six regressors: (1) the trial, (2) the reception of the milkshake solution, (3) the reception of the tasteless solution, (4) water rinsing, (5) the question about solution pleasantness, and (6) intensity. No motion correction regressor was included in the GLM, since we already removed motion components from the signal. Group-level statistical $t$ maps were then created by combining subject-level estimated $\beta$ weights (milkshake $>$ tasteless) and residuals.

A multiple comparisons correction was done using the Analysis of Functional Neurolmages software (AFNl; version 20.2; Cox, 1996). First, we used the 3dFWHMx function with the spatial auto-correlation flag on to estimate the intrinsic spatial smoothness of the noise in our data. The estimate values were averaged across participants and then used in the corrected 3dClustSim function (Cox et al., 2017) to determine, via Monte Carlo simulation of the noise field, a minimum cluster extent corrected for multiple comparisons (at $\alpha=0.05$ ). This guarantees that a group of individual voxels under an uncorrected height threshold of $p<0.001$ with a greater cluster size than the minimum extent would only occur $<5 \%$ of the time.

We report the minimum extent threshold, the cluster's peak MNI coordinates, and the number of consecutive 
A

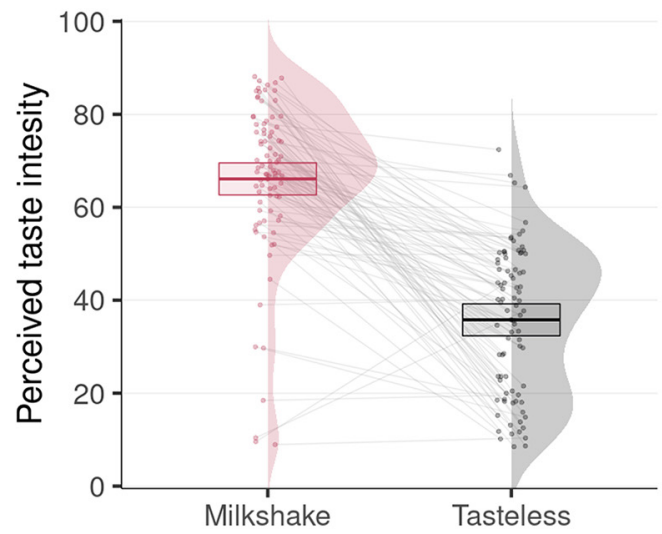

B

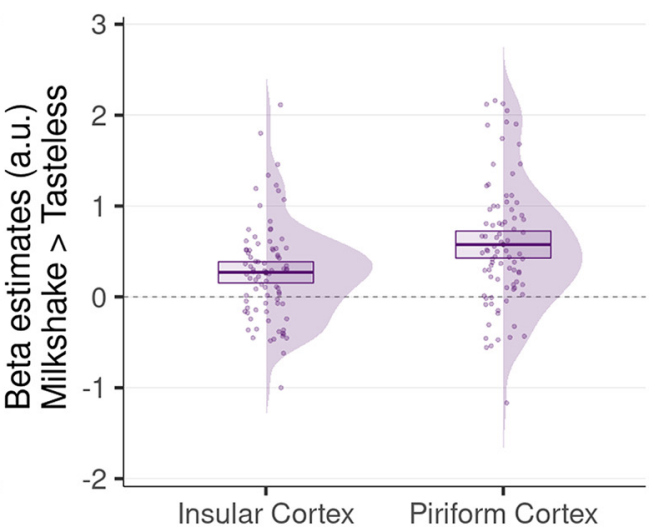

Figure 3. Behavioral and fMRI results. $\boldsymbol{A}$, Individual estimates, densities, and overall mean of perceived taste intensity of the milkshake and the tasteless solutions. $\boldsymbol{B}$, Individual $\beta$ estimates, densities, and overall means of the milkshake $>$ tasteless contrast across participants during taste delivery extracted from voxel clusters within the insular and piriform cortex. Error bars represent $95 \% \mathrm{Cl}(n=85)$.

significant voxels at $p<0.001$ within the cluster $(k)$. Finally, we display the statistical $t$ maps of our group results for the milkshake $>$ tasteless contrast surviving cluster-level correction overlaid on a 3D semi-inflated surface brain template in the MNI space.

\section{Code and data accessibility}

The computer code used to produce the mouthpiece as well as to preprocess and analyze the data are available in a publicly hosted software repository (https://github.com/ munoztd0/Mouthpiecegusto). Unthresholded statistical $t$ maps are available on the Neurovault platform (https:// neurovault.org/images/442236/).

\section{Results}

We analyzed the taste intensity ratings using a paired $t$ test to compare the two conditions (milkshake or tasteless). As expected, participants rated the milkshake solution as significantly more intense than the tasteless solution $\left(\mu=30.33, S E \pm 2.5, t_{(84)}=12.40, p<0.001, d_{z}=\right.$ $1.35,95 \% \mathrm{Cl}=[1.04,1.63], B F_{10}=9.23 \times 10^{20} ;$ see Fig. $3 A$ ).

We report the results from our group-level analysis using a height threshold of $p<0.001$, with a minimum cluster extent threshold corrected for multiple comparisons at $p<0.05$ ( $k=123$ voxels). For the taste reactivity task, the pleasant solution (milkshake $>$ tasteless) activated the primary olfactory (piriform) cortex bilaterally (right: $\mathrm{MNI}[x y z]=[-22-3-14], k=282$; left: $\mathrm{MNI}[x y z]=$ [21 -6 -14], $k=149$ ), the primary gustatory (middle insular) cortex (left: MNI [xyz] = [21 -6-14], $k=149$ ), and the primary somatosensory (postcentral/parietal operculum) cortex (Figs. 3A, 4).

To compute observed power calculations within our two regions of interest, namely the insular and piriform cortex, we extracted the averaged $\beta$ values from within these regions and calculated their standardized effect size $\left(d_{z}\right)$. We report a $d_{z}=0.41$ for the insula and $d_{z}=0.56$ for the piriform cortex and estimate that to reproduce these results at $90 \%$ power and $\alpha=0.05$, then 53 or 29 participants are needed for the insula and the piriform cortex, respectively (details of the power analysis, including power curves, are provided in the Extended Data Fig. 4-2).

\section{Discussion}

In this article, we presented a 3D-printed fMRI-compatible mouthpiece for the study of human taste and flavor perception in MRI settings. After describing this mouthpiece, we reported the results of a 3 Tesla fMRI study providing evidence that this mouthpiece allows us to obtain an effective measure of brain related activity during the consumption of gustatory stimuli.

In this large sample $(n=85)$ study, we demonstrate the effectiveness and validity of our procedure by showing significant clusters of activation within the same regions that have been reported throughout different meta-analyses on taste (Yeung et al., 2017) and olfaction (Seubert et al., 2013). More precisely, we found strong activations of the following: (1) the left middle insular cortex, which has consistently been identified as the human primary gustatory cortex (Buck and Bargmann, 2000; Small and Faurion, 2015), (2) the postcentral/parietal operculum gyrus, which has been reported to be the primary cortex for oral somatosensory representation in humans (Boling et al., 2002), and (3) the anterior medial temporal lobes, including the hippocampal formation and the amygdaloid complex, that have also both been found to play a crucial role in food intake (Davidson et al., 2009; Petrovich, 2011; Coppin, 2016). Our results are also in agreement with an asymmetric model of taste perception (lannilli and Gudziol, 2019), where right-handed populations tend to have stronger left dominance in the insula.

Importantly, we encountered some limitations that should be addressed. First, some participants reported 
L
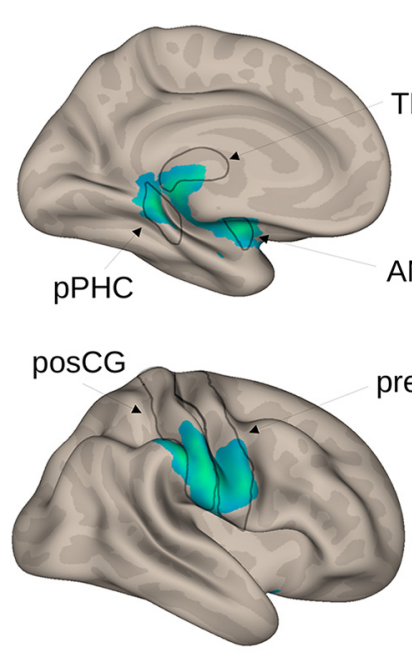

$\mathrm{R}$

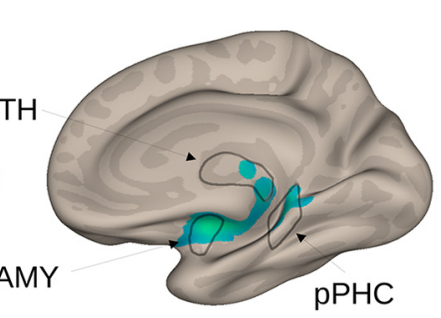

preCG

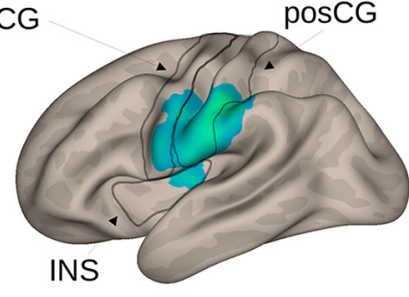

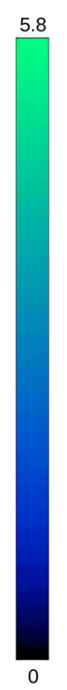

Figure 4. Neural correlates of taste. Regions in which the BOLD signal positively correlates with the magnitude of the contrast milkshake $>$ tasteless $(n=85)$. Statistical $t$ maps are shown with a threshold of $p<0.001$ and a minimum cluster extent threshold (corrected for multiple comparisons) of 123 voxels. Color scale bar shows $t$ statistic values. AMY, amygdala; pPHC, posterior parahippocampal cortex; TH, thalamus; posCG, postcentral gyrus; preCG, precentral gyrus; INS, insula. Detailed results are presented in Extended Data Figures 4-1, 4-2.

that a 40-mm-long mouthpiece was a bit too long and thus, uncomfortable. This can easily be alleviated by printing a shorter mouthpiece in those cases. We also tried to extend our setup to a non-MRI context, where participants would be seated in an upright position. It appeared that the liquids did not flow as consistently and precisely as they did in a lying position, and suggests that the prototype would have to be modified for such contexts. In a few cases and during intensive use, we also noticed that the plastic could become porous, so that the joints between the tubes and the teat were no longer perfectly sealed. As a result, some participants reported that the rinsing liquid had run down their cheeks. However, this did not prevent the stimuli from being sent, but it is something that the researchers should monitor. One option might be to choose a less porous plastic that is still within the country's legislative constraints on plastics permitted for food contact. Moreover, we think it is important to tell participants to place their tongue in such a way as to let the solutions flow without blocking the teat to deliver drops of liquid comfortably and accurately.

Another caveat to the interpretation of our results is that we have not controlled for temperature and mechanosensory information. Existing devices have managed to control for this by having the delivery tubes running through a water bath at a controlled temperature (e.g., $37^{\circ} \mathrm{C}$ ) as well as delivering a continuous spray of the solution over the tongue via a spray head to avoid mechanical simulation effects (lannilli et al., 2015; Andersen et al., 2019). While a spray taste delivery system has proved its efficacy and reliability for event related taste pulses, it could not be used with our test stimuli (i.e., milkshake) because of its high viscosity. This feature was important for our design since we wanted to be able to use realistic feeding paradigms (e.g., milkshakes) as it has been recently advocated by the new "good practice in food-related neuroimaging" (Smeets et al., 2019). We think however that the solutions from lannilli et al. (2015) and Andersen et al. (2019) could be compatible with our mouthpiece. We suggest future investigators that either want to study or to control for the effects of temperature and mechanosensory information on taste perception to take these methods into account.

Additionally, we unfortunately could not provide a direct comparison between the data collected with our new design and data collected from other commonly used mouthpieces. This will hopefully be possible in future investigations through an ever-increasing number of data sharing initiatives.

To conclude, the main advantages of this mouthpiece are its low cost, flexibility, ease to produce and fMRIcompatible design. Any lab with access to a 3D printer can make one or could otherwise get them made by any 3D printing service company since our plans are freely available. But most importantly, it is flexible and can be modified for any particular case. It can easily comply with different countries' sanitary regulations or be adjusted for different types of liquid or viscosity levels. It also does not require any modification to any preexisting apparatus and will integrate to most gustometer setups without any additional work.

More theoretically, affective neuroscience could benefit from the inclusion of more studies in olfaction and taste using primary rewards. This could provide the means for an integrative approach to study the emotional nature of reward (Nummenmaa and Sander, 2020). We think that this new method could help promote the use of primary rewards (e.g., milkshakes) instead of more widely used food pictures to measure hedonic processes. This is extremely important because, not only does it allow a direct comparison to be drawn with the animal literature on 
innate food rewards, but it also helps avoid reward type-dependent neural circuits of secondary rewards (Sescousse et al., 2013; Nakamura et al., 2020). Moreover, taste consumption can induce an affective experience in itself rather than a representation of the affective experience (i.e., pictures of food), which is a crucial property to properly study reward processing (Pool et al., 2016).

\section{References}

Andersen CA, Alfine L, Ohla K, Höchenberger R (2019) A new gustometer: Template for the construction of a portable and modular stimulator for taste and lingual touch. Behav Res 51:2733-2747.

Avants BB, Tustison N, Song G, Cook P, Klein A, Gee JC (2011) Advanced normalization tools (ANTS). Neurolmage 54:2033-2044.

Bartoshuk LM, McBurney DH, Pfaffmann C (1964) Taste of sodium chloride solutions after adaptation to sodium chloride: Implications for the "water taste". Science 143:967-968.

Beckmann CF, Smith SM (2004) Probabilistic independent component analysis for functional magnetic resonance imaging. IEEE Trans Med Imaging 23:137-152.

Boling W, Reutens DC, Olivier A (2002) Functional topography of the low postcentral area. J Neurosurg 97:388-395.

Brainard DH (1997) The psychophysics toolbox. Spat Vis 10:433436.

Breiman L (2001) Random forests. Mach Learn 45:5-32.

Buck LB, Bargmann Cl (2000) Smell and taste: The chemical senses. In: Principles of neural science (Kandel ER and Schwartz JH, eds), pp 712-734. New York: McGraw-Hill.

Canna A, Prinster A, Fratello M, Puglia L, Magliulo M, Cantone E, Pirozzi MA, Di Salle F, Esposito F (2019) A low-cost open-architecture taste delivery system for gustatory $\mathrm{fMRI}$ and $\mathrm{BCl}$ experiments. J Neurosci Methods 311:1-12.

Conn R, Kolstad J, Borzelleca J, Dixler D, Filer L Jr, LaDu B Jr, Pariza $M(1995)$ Safety assessment of polylactide (PLA) for use as a foodcontact polymer. Food Chem Toxicol 33:273-283.

Coppin G (2016) The anterior medial temporal lobes: Their role in food intake and body weight regulation. Physiol Behav 167:60-70.

Coppin G (2020) The COVID-19 may help enlightening how emotional food is. Science Food NPJ 4:1-3.

Cox RW (1996) AFNI: Software for analysis and visualization of functional magnetic resonance neuroimages. Comput Biomed Res 29:162-173.

Cox RW, Chen G, Glen DR, Reynolds RC, Taylor PA (2017) fMRI clustering and false-positive rates. Proc Natl Acad Sci USA 114: E3370-E3371.

Davidson TL, Chan K, Jarrard LE, Kanoski SE, Clegg DJ, Benoit SC (2009) Contributions of the hippocampus and medial prefrontal cortex to energy and body weight regulation. Hippocampus 19:235-252

Goto TK, Yeung AWK, Suen JLK, Fong BSK, Ninomiya Y (2015) High resolution time-intensity recording with synchronized solution delivery system for the human dynamic taste perception. J Neurosci Methods 245:147-155.

Griffanti L, Douaud G, Bijsterbosch J, Evangelisti S, Alfaro-Almagro F, Glasser MF, Duff EP, Fitzgibbon S, Westphal R, Carone D, Beckmann CF, Smith SM (2017) Hand classification of fMRI ICA noise components. Neurolmage 154:188-205.

Holmes A, Friston K (1988) Generalisability, random effects and population inference. Neurolmage 7:1-3.
Iannilli E, Gudziol V (2019) Gustatory pathway in humans: A review of models of taste perception and their potential lateralization. J Neurosci Res 97:230-240.

lannilli E, Beger M, Fürer R, Hummel T (2015) A gustatory stimulator. J Neurosci Methods 255:12-16.

Jenkinson M, Beckmann CF, Behrens TEJ, Woolrich MW, Smith SM (2012) FSL. Neurolmage 62:782-790.

Lakens D (2013) Calculating and reporting effect sizes to facilitate cumulative science: A practical primer for t-tests and ANOVAs. Front Psychol 4:863-864.

Morey RD, Rouder JN, Jamil T (2015) BayesFactor: Computation of bayes factors for common designs. $\mathrm{R}$ package, version 0.9 .

Mumford JA, Poldrack RA (2007) Modeling group fMRI data. Soc Cogn Affect Neurosci 2:251-257.

Nakamura Y, Imafuku M, Nakatani H, Nishida A, Koike S (2020) Difference in neural reactivity to taste stimuli and visual food stimuli in neural circuits of ingestive behavior. Brain Imaging Behav 14:1395-1405.

Nummenmaa L, Sander D (2020) Reward and emotion in the brain. psyArXiv:10.31234.

O'Doherty JP, Deichmann R, Critchley HD, Dolan RJ (2002) Neural responses during anticipation of a primary taste reward. Neuron 33:815-826.

Penny WD, Friston KJ, Ashburner JT, Kiebel SJ, Nichols TE (2011) Statistical parametric mapping: The analysis of functional brain images. Amsterdam: Elsevier.

Petrovich GD (2011) Forebrain circuits and control of feeding by learned cues. Neurobiol Learn Mem 95:152-158.

Pool E, Sennwald V, Delplanque S, Brosch T, Sander D (2016) Measuring wanting and liking from animals to humans: A systematic review. Neurosci Biobehav Rev 63:124-142.

Pruim RH, Mennes M, Buitelaar JK, Beckmann CF (2015) Evaluation of ICA-AROMA and alternative strategies for motion artifact removal in resting state fMRI. Neuroimage 112:278-287.

R Core Team (2019) R: A language and environment for statistical computing. Vienna: R Foundation for Statistical Computing.

Salimi-Khorshidi G, Douaud G, Beckmann CF, Glasser MF, Griffanti L, Smith SM (2014) Automatic denoising of functional MRI data: Combining independent component analysis and hierarchical fusion of classifiers. Neurolmage 90:449-468.

Sescousse G, Caldú X, Segura B, Dreher J-C (2013) Processing of primary and secondary rewards: A quantitative meta-analysis and review of human functional neuroimaging studies. Neurosci Biobehav Rev 37:681-696.

Seubert J, Freiherr J, Djordjevic J, Lundström JN (2013) Statistical localization of human olfactory cortex. Neurolmage 66:333-342.

Small DM, Faurion A (2015) Mapping brain activity in response to taste stimulation. In: Handbook of olfaction and gustation (Doty R, ed), pp 775-794. Hoboken: Wiley.

Small DM, Gregory MD, Mak YE, Gitelman D, Mesulam MM, Parrish T (2003) Dissociation of neural representation of intensity and affective valuation in human gustation. Neuron 39:701-711.

Smeets PA, Dagher A, Hare TA, Kullmann S, van der Laan LN, Poldrack RA, Preissl H, Small D, Stice E, Veldhuizen MG (2019) Good practice in food-related neuroimaging. Am J Clin Nutr 109:491-503.

Talmi D, Seymour B, Dayan P, Dolan RJ (2008) Human Pavlovian-instrumental transfer. J Neurosci 28:360-368.

Yeung AWK, Goto TK, Leung WK (2017) Basic taste processing recruits bilateral anteroventral and middle dorsal insulae: An activation likelihood estimation meta-analysis of $\mathrm{fMRI}$ studies. Brain Behav 7:e00655. 